\title{
Osteogenic potential of bone marrow stromal cells derived from streptozotocin-induced diabetic rats
}

\author{
YAN-FANG ZHAO $^{1}$, DE-LIANG ZENG ${ }^{1}$, LUN-GUO XIA $^{2}$, SONG-MEI ZHANG ${ }^{1}$, \\ LIAN-YI XU ${ }^{1}$, XIN-QUAN JIANG ${ }^{1,3}$ and FU-QIANG ZHANG ${ }^{1}$
}

\begin{abstract}
Departments of ${ }^{1}$ Prosthodontics and ${ }^{2}$ Oral and Maxillofacial Surgery, ${ }^{3}$ Oral Bioengineering Laboratory/Regenerative Medicine Laboratory, Shanghai Research Institute of Stomatology, Shanghai Key Laboratory of Stomatology, Ninth People's Hospital affiliated to Shanghai Jiao Tong University School of Medicine, Shanghai, P.R. China
\end{abstract}

Received September 29, 2012; Accepted November 9, 2012

DOI: $10.3892 / \mathrm{ijmm} .2013 .1227$

\begin{abstract}
Type 1 diabetes mellitus (T1DM) is associated with a series of bone complications, which are still a great challenge in the clinic. Bone marrow stromal cells (BMSCs) are crucial to bone remodeling and are attractive candidates for tissue engineering. Hence, we aimed to investigate whether impaired functions of BMSCs play a role in the pathogenesis of bone complications associated with T1DM. BMSCs were isolated from normal and streptozotocin-induced diabetic rats, and their proliferation and osteogenic differentiation ability were analyzed. Diabetic BMSCs demonstrated reduced proliferation ability, osteoblast gene expression, alkaline phosphatase activity and mineralization. Nude mice transplanted with diabetic BMSCs in a calcium phosphate cement scaffold exhibited reduced new bone formation, as detected by hematoxylin and eosin staining and immunohistochemistry. These changes may be partially related to impaired insulin and insulin-like growth factor 1 (IGF-1) signaling. Weak gene expression of insulin receptor (IR), IGF-1, insulin-like growth factor 1 receptor (IGF-1R), and insulin receptor substrate-1 (IRS-1) was observed in the diabetic BMSCs compared with normal BMSCs, together with decreased protein level of IGF-1, IGF-1R, IRS-1 and phosphorylated extracellular signal-regu-
\end{abstract}

Correspondence to: Professor Fu-Qiang Zhang, Department of Prosthodontics, Ninth People's Hospital affiliated to Shanghai Jiao Tong University School of Medicine, 639 Zhizaoju Road, Shanghai 200011, P.R. China

E-mail: fredzc@online.sh.cn

Professor Xin-Quan Jiang, Oral Bioengineering Laboratory/ Regenerative Medicine Laboratory, Shanghai Research Institute of Stomatology, Department of Prosthodontics, Shanghai Key Laboratory of Stomatology, Ninth People's Hospital affiliated to Shanghai Jiao Tong University School of Medicine, 639 Zhizaoju Road, Shanghai 200011, P.R. China

E-mail:xinquanj@yahoo.cn

Key words: diabetes mellitus, bone marrow stromal cells, osteogenesis, tissue engineering, insulin, insulin-like growth factor 1 lated kinase. Therefore, impaired proliferation and osteogenic potential of BMSCs may be responsible for bone complications related to T1DM, mediated partially by impaired insulin and IGF-1 signaling. These findings may provide a new target with which to devise strategies for therapy.

\section{Introduction}

Type 1 diabetes mellitus (T1DM) is an increasingly prevalent systemic disease in the world. It is well known that this disease has detrimental effects on bone remodeling, leading to a series of bone complications including osteopenia, osteoporosis, increased risk of fracture, as well as impaired bone healing and regeneration (1-3). All of the above problems may significantly affect the quality of life of patients. Despite numerous studies conducted, to date, little is known about the detailed pathogenesis of these effects of diabetes, and few effective therapies exist for healing these bone disorders (4).

Bone marrow stromal cells (BMSCs) are a major source of osteoblasts and are crucial for bone remodeling and repair through direct regeneration such as cell differentiation or maturation, or indirect mechanisms via paracrine effects such as increased vascularization (5). In addition, due to their easy availability and multipotent potential, BMSCs are an attractive candidate for tissue engineering applications, which have been successfully used to enhance bone repair $(6,7)$. Previous studies have demonstrated that diabetes has direct detrimental effects on many types of cells, including impaired proliferation and function of adipose tissue resident mesenchymal stem cells and bone mesenchymal stem cells (8-10). Recently, impairments of BMSCs were reported to be responsible for osteopenia in diabetes which might represent a cellular mechanism (11). However, to date, the effects of diabetes on the osteogenic potential of BMSCs and the possible related mechanisms remain unclear.

T1DM is characterized by hypoinsulinemia and insulinlike growth factor 1 (IGF-1) deficiency. Both insulin and IGF-1 are important for the normal development of mineralized skeleton and bone remodeling. Moreover, the imbalance between insulin and the IGF system may contribute to the bone disorders in diabetes $(12,13)$. In particular, delivery of insulin or IGF-1 enhanced bone repair in diabetic rats $(14,15)$. 
Insulin and IGF-1 may function in the process of bone formation through insulin receptor (IR), IGF-1 receptor (IGF-1R) and a common hybrid receptor, which could activate the insulin receptor substrate (IRS) proteins that regulate a variety of signaling pathways controlling cell proliferation, differentiation, apoptosis and metabolism (16). In vitro, insulin and IGF-1 promote osteoblasts to express IR or IGF-1R, and enhance cell proliferation and differentiation via the extracellular signal-regulated kinase (ERK) pathway $(17,18)$. Conversely, mice lacking IR in osteoblasts resulted in reduced trabecular bone and mineralization (19). Mice lacking IGF-1R in osteoblasts or pre-osteoblastic cells also showed decreased bone mass and mineral deposition rates $(20,21)$. Whether abnormalities in insulin and IGF-1 signaling may explain altered osteogenic potential of BMSCs derived from diabetic rats has not been determined.

Therefore, in the present study, we aimed to investigate and compare osteoblastic differentiation of BMSCs derived from diabetic rats in vitro, and bone formation capacity in vivo of BMSCs derived from normal and diabetic rats. We also determined whether alterations in osteogenic potential of diabetic BMSCs are related to parallel decreases in expression of IGF-1, IGF-1R, IR and IRS-1 via the ERK signaling pathway. Systematic evaluation of these questions may provide a comprehensive understanding of bone complications associated with diabetes and the possible relevance for future cell therapies for these diseases.

\section{Materials and methods}

Animal models. All animal experiments were approved by the Animal Research Committee of the Ninth People's Hospital affiliated to Shanghai Jiao Tong University, School of Medicine. Sixteen 4-week-old male Wistar rats and 4 4-weekold male nude Balb/c mice were used in the study. Rats were randomly divided into 2 groups. Diabetes was induced in 8 rats via a single intraperitoneal injection of streptozotocin (65 mg/kg) (Sigma, St. Louis, MO, USA) dissolved in $0.01 \mathrm{M}$ citrate buffer ( $\mathrm{pH} 4.5$ ). Another 8 age-matched normal rats that received no injection served as the controls. Diabetes in the rats was confirmed when blood glucose concentrations were higher than $16.7 \mathrm{mmol} / \mathrm{l}$ tested by a blood glucose meter (Accu-Chek Performa; Roche Diagnostics, Indianapolis, IN, USA) 1 week post-injection (22). The blood glucose concentrations were tested on the same day before injection, 1, 4, 8 and 12 weeks post-injection. Body weights were measured on the same day before injection, 1 and 12 weeks post-injection. All animals were fed a regular diet and given water ad libitum.

Isolation and culture of rat BMSCs. After euthanasia, BMSCs were harvested from both the tibia and femur bone marrow of the normal and diabetic rats, and cultured in low glucose Dulbecco's modified Eagle's medium (DMEM, low glucose; Gibco-BRL, Grand Island, NY, USA) supplemented with $10 \%$ fetal bovine serum (Hyclone, Logan, UT, USA), containing $100 \mathrm{U} / \mathrm{ml}$ penicillin, $100 \mathrm{U} / \mathrm{ml}$ streptomycin and 2 mM L-glutamine (Sigma) as previously described (23). After BMSCs were incubated for $24 \mathrm{~h}$, the medium was changed to discard non-adherent cells. Then the medium was refreshed every 3 days. When confluence reached $\sim 80 \%$, cells were passaged for expansion. The following experiments used cells at passage 2 or 3 . To study the osteoblastic differentiation of BMSCs, osteogenic medium contained 10-8 M dexamethasone, $50 \mu \mathrm{g} / \mathrm{ml} \mathrm{L-2-ascorbic} \mathrm{acid} \mathrm{and} 10 \mathrm{mM}$ $\beta$-glycerophosphate were used.

Cell proliferation assay. To measure the proliferation of cells, MTT assay was used to assess the amount of viable cells (24). Briefly, cells were seeded at a density of $5 \times 10^{3}$ cells/well on a 96-well plate. On the next day, the medium was replaced by fresh medium, and cells were cultured for 1, 3, 5 and 7 days. MTT $(5 \mathrm{mg} / \mathrm{ml})$ was added into each well and incubated for $4 \mathrm{~h}$. After removing the medium, DMSO was used to dissolve the formazan crystals and the absorbance value was measured using a microplate reader (Bio-Tek, Winooski, VT, USA) at $490 \mathrm{~nm}$.

Real-time PCR analysis. Total RNA was extracted at day 7 using TRIzol reagent (Invitrogen, Carlsbad, CA, USA) and was transcribed with PrimeScript RT reagent kit (Takara, Kyoto, Japan). cDNA amplification and detection were performed using the Bio-Rad iQ5 real-time PCR system (Bio-Rad, Hercules, CA, USA) using SYBR Premix Ex Taq kit (Takara) and specific primers (Table I). The relative gene expression level was normalized to the internal control $\left(\beta\right.$-actin) based on the $2^{-\Delta \Delta \mathrm{Ct}}$ method.

ALP staining and ALP activity assay. For ALP staining, after being fixed for $15 \mathrm{~min}$ at $4^{\circ} \mathrm{C}$, BMSCs at days 7 and 14 in osteogenic medium were treated with a BCIP/NBT solution (Beyotime, Shanghai, China) in the dark, and areas that stained purple were regarded as positive. ALP activities were determined using p-nitrophenyl phosphate (Sigma) as described previously (25).

Alizarin red staining for mineralization measurement. After culturing in the osteogenic medium for 21 days, BMSCs were fixed in $70 \%$ ethanol and stained with $40 \mathrm{mM}$ Alizarin Red S solution. Then the stain was desorbed with $10 \%$ cetylpyridinium chloride (Sigma) for $1 \mathrm{~h}$, and the Alizarin Red concentration of dye extracts was determined by absorbance at $590 \mathrm{~nm}$ (Bio-Tek).

Western blot analysis. For western blotting, cells at day 7 were lysed with a protein extraction reagent containing protease inhibitor cocktail, phosphatase inhibitor cocktail and phenylmethanesulfonyl fluoride (PMSF) (Kangchen, Shanghai, China). The protein concentration was measured according to the BCA protein assay kit manufacturer's protocol (Beyotime). Equal protein samples were separated on SDS-polyacrylamide gel electrophoresis (PAGE) and then electro-transferred to polyvinylidene difluoride membranes. The membranes were blocked with primary antibodies: rabbit anti-rat IGF-1R, rabbit anti-rat IRS-1 (both from Bioworld), rabbit anti-rat ERK, rabbit anti-rat phosphorylated-ERK (both from Cell Signaling Technology, Inc.) and mouse anti-rat $\beta$-actin (Sigma) and incubated. Finally, the blots were visualized with horseradish peroxidase (HRP)-conjugated goat anti-rabbit or anti-mouse IgG (Beyotime) using the ECL Plus reagents (Amersham 
Table I. Sequences of the primers used for real-time PCR.

\begin{tabular}{lll}
\hline Genes & \multicolumn{1}{c}{ Forward primer } & \multicolumn{1}{c}{ Reverse primer } \\
\hline$\beta$-actin & CACCCGCGAGTACAACCTTC & CCCATACCCACCATCACACC \\
Runx2 & ATCCAGCCACCTTCACTTACACC & GGGACCATTGGGAACTGATAG \\
OCN & GCCCTGACTGCATTCTGCCTCT & TCACCACCTTACTGCCCTCCTG \\
ALP & GGCTCTGCCGTTGTTTC & GGGTTGGGTTGAGGGACT \\
IR & ACATTGCCCTGAAGACCAAC & AACAGCATGAATCCCAGGAG \\
IGF-1 & CCGCTGAAGCCTACAAAGTC & GGGAGGCTCCTCCTACATTC \\
IGF-1R & GTGCTGTACGCCTCTGTGAA & TTGCAGCCTCATTCACTGTC \\
IRS-1 & TGTGCCAAGCAACAAGAAAG & ACGGTTTCAGAGCAGAGGAA
\end{tabular}

Pharmacia Biotech, Arlington Heights, IL, USA) using UVItec Alliance 4.7 gel imaging system. The relative integrated density of each protein band was analyzed by NIH image $1.34 \mathrm{~s}$.

Surgical implantation of BMSCs. The porous calcium phosphate cement (CPC) scaffolds (4-mm diameter, 2-mm height) used in the study were purchased from Rebone Biomaterial Co., Ltd. (Shanghai, China). Average diameter of the scaffold pores was $400 \mu \mathrm{m}$ and average porosity was $70 \%$. BMSCs from diabetic or normal rats were seeded at a density of $2 \times 10^{7}$ cells $/ \mathrm{ml}$ in $20 \mu \mathrm{l}$ on the CPC scaffolds. The implants were then subcutaneously implanted into 4 nude mice at the intrascapular area. Each mouse received the following 3 groups of complexes: CPC scaffold group $(n=4)$, CPC/ normal BMSCs $(n=4)$ and CPC/diabetic BMSCs $(n=4)$.

Histological and immunohistochemical analysis. Four weeks after surgery, the implants were harvested, fixed, decalcified, embedded in paraffin, sectioned $(4-\mu \mathrm{m})$ and stained with hematoxylin and eosin (H\&E). Photomicrographs of each section were captured with a light microscope (Olympus, Tokyo, Japan) and histomorphological analysis was performed using the automated image analysis system, Image Pro 5.0 (Media Cybernetics, Silver Spring, MD, USA). The percentages of new bone area were calculated by the mean value of the 3 parallel sections randomly selected from serial sections of each sample cut at cross-sectional directions. The mean value of the 3 measurements was calculated for each implant, and were further used to calculate mean values for each group. New bone formation in each section was defined as the percentage of new bone area in the observed areas/the entire implant (26).

Immunohistochemical analyses were performed as described previously (27). Briefly, the paraffin sections were dewaxed and rehydrated, then immersed in $\mathrm{H}_{2} \mathrm{O}_{2}$ to quench peroxidase. After blocking, the sections were incubated with primary mouse monoclonal antibodies against OCN (Abcam, Cambridge, UK) overnight at $4^{\circ} \mathrm{C}$. Then the sections were incubated with HRP-conjugated rabbit anti-mouse IgG (Beyotime) for $1 \mathrm{~h}$ at room temperature. Finally, a brown color was produced by using the diaminobenzidine (DAB; Beyotime, Jiangsu, China) kit and sections were counter- stained with hematoxylin. Photomicrographs of each section were captured with a light microscope (Olympus).

Statistical analysis. Results are expressed as means \pm standard deviation (SD). Comparisons were performed by independent samples t-test using SPSS 11.0 (SPSS, Inc., Chicago, IL, USA), and $\mathrm{P}<0.05$ was considered to indicate a statistically significant result.

\section{Results}

Experimental animal model. Rats treated with streptozotocin showed typical symptoms of diabetes: high intake of food, polydipsia and polyuria. Compared with normal rats, the diabetic rats exhibited significantly higher blood glucose levels (Fig. 1a) and lower body weights (Fig. 1b).

Cell proliferation. Proliferation of the diabetic BMSCs proceeded slower than the normal BMSCs, with no significant difference at day 1 and a significant difference at days 3,5 and 7 (Fig. 2).

Real-time PCR analysis. The gene expression of osteogenic differentiation markers, ALP, Runx2 and OCN, was evaluated at day 7 after cells were incubated in osteogenic medium. The mRNA levels of ALP, Runx 2 and OCN in the diabetic BMSCs were significantly reduced to $\sim 39,38$ and $2 \%$ of the control level, respectively (Fig. 3).

ALP activity and Alizarin Red assay. At days 7 and 14 after culture in osteogenic medium, diabetic BMSCs demonstrated weaker ALP-positive staining compared with the normal BMSCs (Fig. 4). Accordingly, the ALP activity in the diabetic BMSCs significantly decreased to $\sim 66$ and $70 \%$ of the normal group, respectively (Fig. 4). Furthermore, Alizarin Red staining, as well as the quantitative analysis of mineralization at day 21 , indicated significantly lower calcium deposition in diabetic BMSCs compared with the normal BMSCs (Fig. 4).

In vivo osteogenic potential of normal BMSCs and diabetic BMSCs. At 4 weeks after implantation, no new bone formation was noted in the CPC scaffold alone group (data not shown), whereas less new bone formation was observed 
a

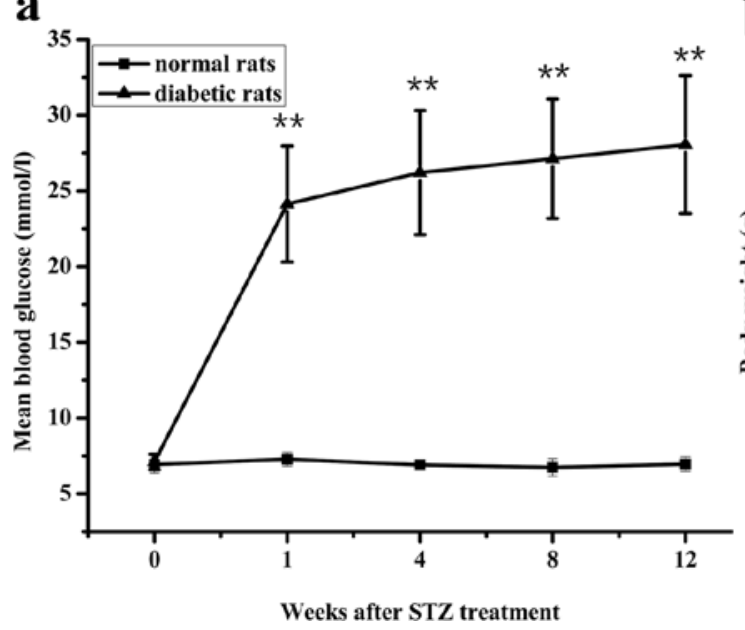

b

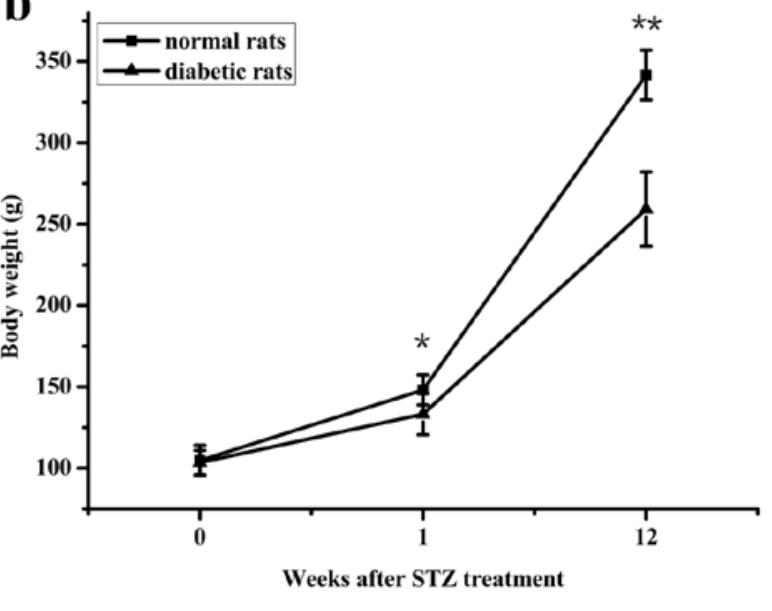

Figure 1. Changes in blood glucose levels and body weights of normal and diabetic rats during the experiment period. (a) Blood glucose was measured on the same day before injection, 1, 4, 8 and 12 weeks post injection. (b) Body weight was measured on the same day before injection, 1 and 12 weeks post injection. $\left(\mathrm{n}=8,{ }^{*} \mathrm{P}<0.01,{ }^{*} \mathrm{P}<0.05\right.$ vs. normal rats).

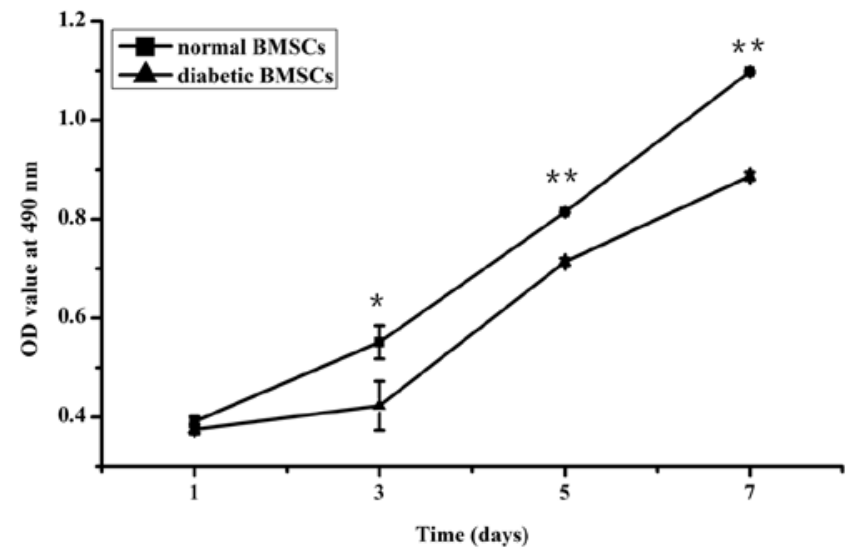

Figure 2. Cell proliferation assay. MTT assay of normal or diabetic BMSCs cultured for $1,3,5$ and 7 days. ${ }^{(* *} \mathrm{P}<0.01,{ }^{*} \mathrm{P}<0.05$ vs. normal BMSCs).

in the CPC/diabetic BMSC group compared with the $\mathrm{CPC} /$ normal BMSC group. Histomorphometrical analysis further revealed that the percentage of new bone area in the $\mathrm{CPC} /$ diabetic BMSC group decreased to $71 \%$ of the normal value (Fig. 5). Likewise, the immunohistochemical staining of OCN displayed less intense staining in the diabetic group compared to the normal group. Collectively, these in vivo data further supplement the in vitro data indicating that the osteogenic potential of diabetic BMSCs are impaired compared with normal BMSCs.

Gene expression and protein levels involved in the insulin and IGF-1 signaling pathway. A significant decrease in IR, IGF-1, IGF-1R and IRS-1 gene expression was observed in diabetic BMSCs compared to normal BMSCs (Fig. 6). Similarly, the protein levels of IGF-IR and IRS-1 significantly decreased in the diabetic BMSCs compared with normal BMSCs (Fig. 6). In addition, the decreased amount of phosphorylated extracellular signal-regulated kinase (p-ERK) protein was detected in diabetic BMSCs compared with control BMSCs, while there

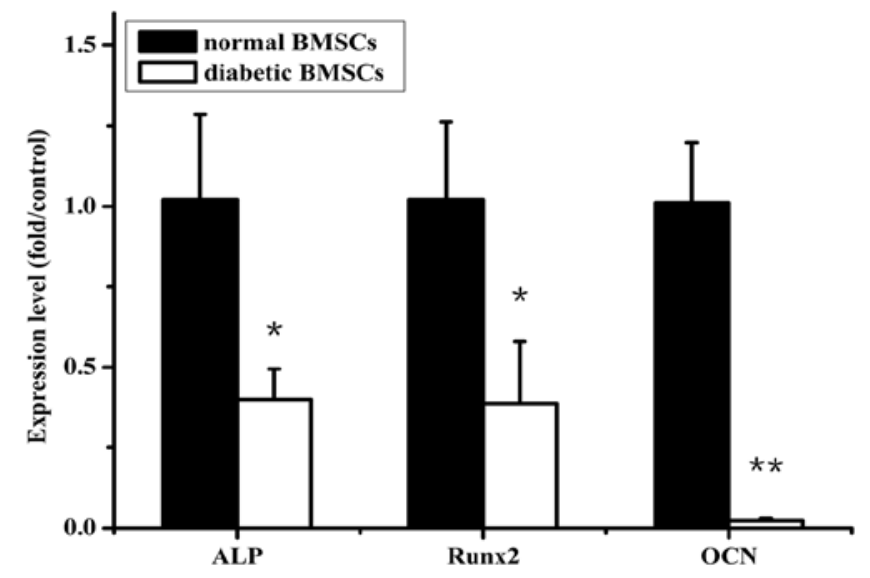

Figure 3. Real-time PCR analysis of expression of osteogenic differentiation genes in normal and diabetic BMSCs at day 7. ALP, Runx 2 and OCN expression was significantly reduced in diabetic BMSCs compared with normal BMSCs. $\left({ }^{* *} \mathrm{P}<0.01,{ }^{*} \mathrm{P}<0.05\right.$ vs. normal BMSCs).

was no significant difference in total ERK expression between diabetic and normal BMSCs (Fig. 6).

\section{Discussion}

It is well documented that diabetes is associated with a series of bone disorders. Previous research has demonstrated that these disorders are primarily due to a defect in bone formation rather than resorption (28). Moreover, previous studies have focused on the dysfunction of osteoblasts, and mainly attributed the defect in bone formation to suppressed differentiation, proliferation and/or bone-forming capacity of osteoblast cells (28). BMSCs mainly reside in the bone marrow and differentiate into osteoblasts, chondrocytes and many types of cells. They also play an important role in bone remodeling and regeneration. However, investigations concerning the role of alterations in BMSCs in bone disorders associated with diabetes are scare. 
a

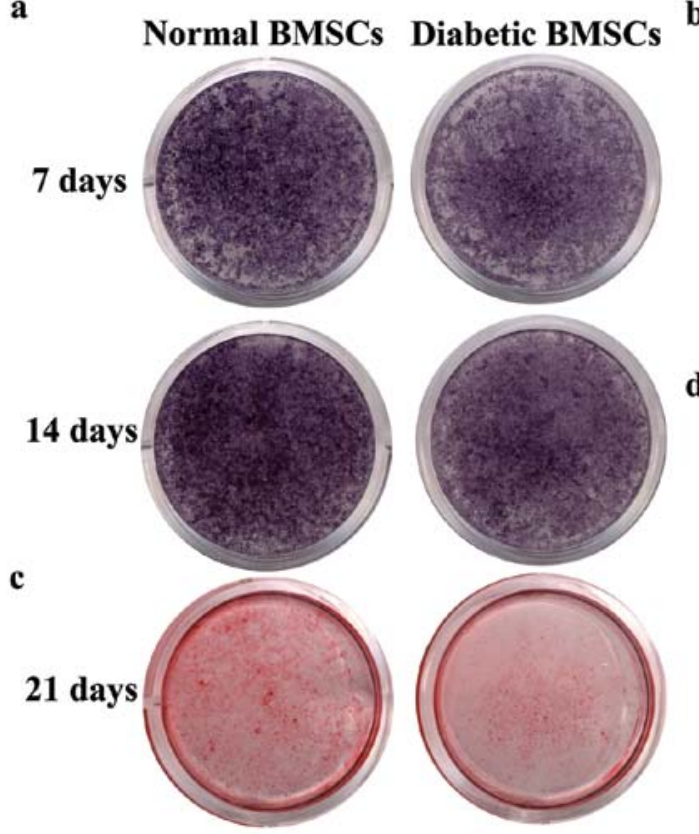

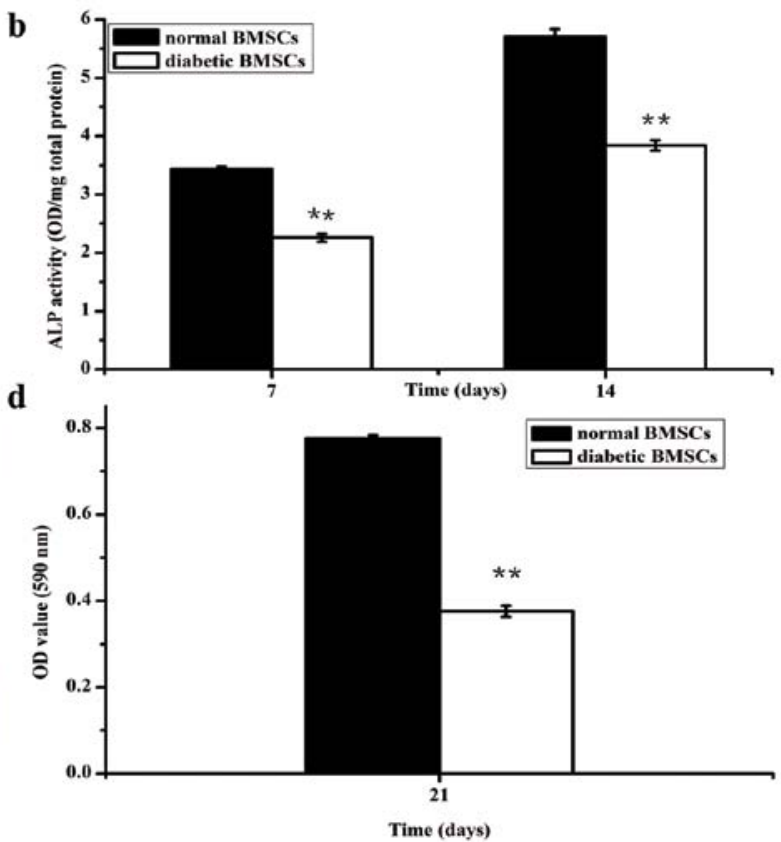

Figure 4. ALP activity and calcium deposit assays. (a) ALP staining and (b) ALP activity of normal and diabetic BMSCs cultured in osteogenic medium at days 7 and 14. (c) Alizarin Red S staining and (d) quantitative mineral deposition of mineralized calcium nodules from normal or diabetic BMSCs cultured in osteogenic medium at day 21 . The ALP activity and quantitative mineral deposition for mineralized calcium nodules were significantly downregulated in diabetic BMSCs compared to normal BMSCs. ("P<0.01 vs. normal BMSCs).
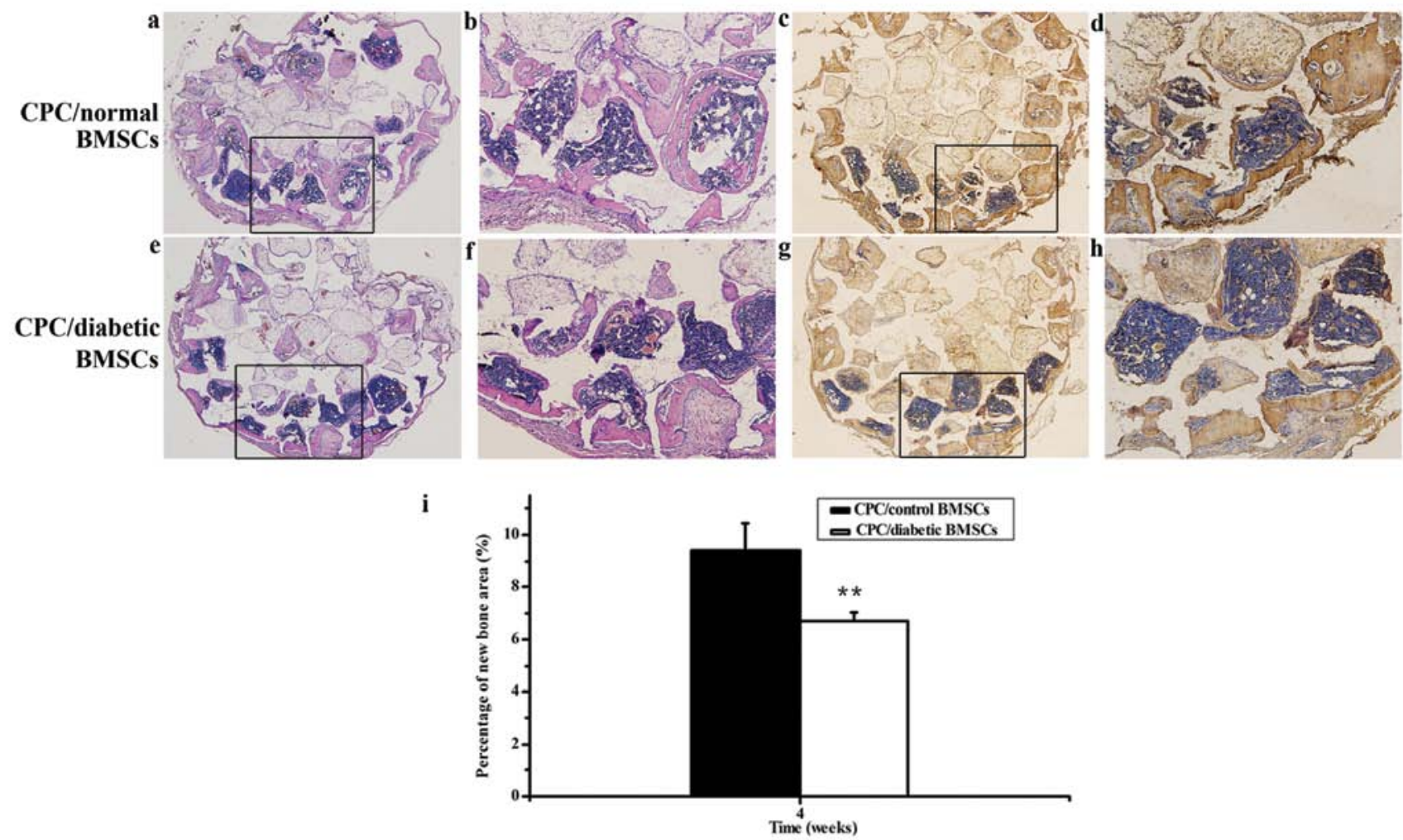

Figure 5. Histological and immunohistochemical findings at 4 weeks after implantation. Newly formed bone was found in (a and b) the CPC/normal BMSC group and (e and f) the CPC/diabetic BMSC group. Immunohistochemical staining for OCN protein in (c and d) the CPC/normal BMSC group and ( $\mathrm{g}$ and $\mathrm{h}$ ) the CPC/diabetic BMSC group. The percentage of new bone area was lower in the CPC/diabetic BMSC group compared to the CPC/normal BMSC group by (i) histomorphometric analysis. (a, c, e, g: x40; b, d, f, g: x100, n=4; "P<0.05 vs. normal BMSCs).

In this study, we studied the proliferation and osteogenic potential of BMSCs derived from diabetic rats. Our results demonstrated that there is a decline in proliferation of diabetic
BMSCs compared with normal controls, consistent with a previous study (10). Subsequently, we studied the osteoblastic differentiation of BMSCs in vitro. Real-time PCR was 


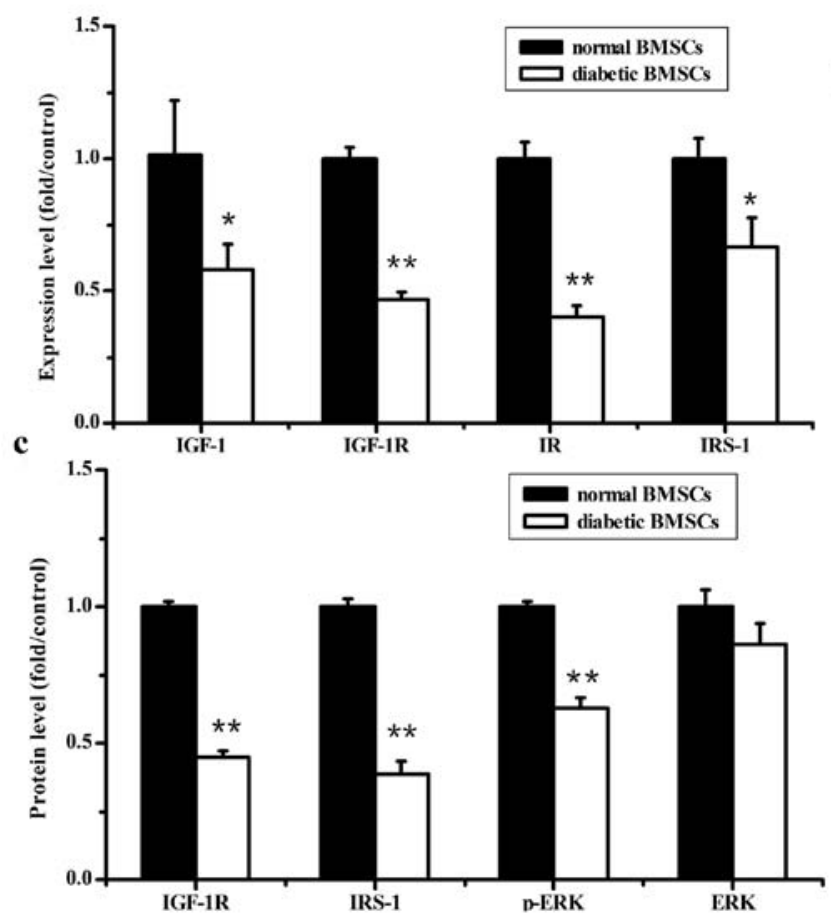

b

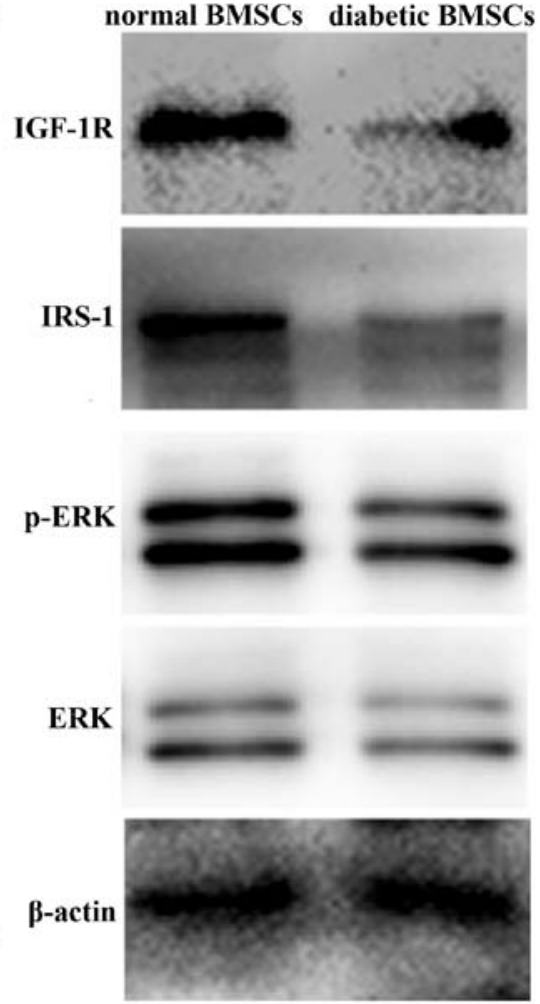

Figure 6. Gene expression and protein levels involved in the insulin and IGF-1 signaling pathway in normal and diabetic BMSCs. (a) mRNA levels of IR, IGF-1, IGF-1R and IRS-1, (b) western blot analyses of IGF-1, IGF-1R, IRS-1, p-ERK and ERK. One representative image from 3 independently performed experiments is shown. (c) Levels of IGF-1, IGF-1R, IRS-1, p-ERK and ERK were quantified by densitometry and expressed graphically $\left({ }^{* *} \mathrm{P}<0.01\right.$, ${ }^{*} \mathrm{P}<0.05$ vs. normal BMSCs).

conducted to detect the mRNA expression of osteogenic markers, ALP, Runx 2 and OCN, in normal and diabetic BMSCs. ALP, a membrane bound enzyme, is a marker of early bone differentiation. Runx 2 is a key transcriptional regulator of osteoblastic differentiation and its expression is essential for normal bone formation. OCN, a marker of late-stage osteoblast differentiation, is a product of osteoblasts and accumulates in the extracellular matrix of bone. As shown in the present study, these osteogenic markers, as well as alkaline phosphatase activity and mineralization were significantly reduced in diabetic BMSCs compared with normal BMSCs. Our data clearly reveal impaired osteogenic potential of diabetic BMSCs, which helps to explain the welldocumented correlation between the incidence of diabetes and bone disorders. It also suggests that BMSCs could be a potential target in the treatment of these disorders.

To investigate the bone regenerative ability of diabetic BMSCs in vivo, we used a well-established ectopic bone formation model. In vivo implantation experiments were carried out for the first time, in which osteogenic differentiation of diabetic BMSCs in vitro was recapitulated in vivo. In nude mice, both types of BMSCs underwent osteoblastic differentiation and formed new bone. Histomorphological analysis demonstrated that the new bone area from the diabetic BMSCs was reduced to $71 \%$ of the normal controls. BMSCs are attractive cell sources for bone tissue engineering, which is effective to enhance bone repair. These results further confirmed the impaired osteogenic potential of diabetic BMSCs, and demonstrated a possibility of cell-based therapy in bone disorders correlated with diabetes, but efforts to compensate for their deficiency should be considered.

Better understanding of the underlying mechanism of diabetes on the osteogenic potential of BMSCs may be helpful to devise successful strategies for therapy. Previous studies have shown that hyperglycemia is an important cause of bone disorder in diabetes (3). In this study, we showed impaired osteogenic potential of diabetic BMSCs in a low glucose culture environment suggesting that the impairment of BMSCs may be durable, and the underlying reasons are not only related to hyperglycemia.

T1DM is also characterized by hypoinsulinemia and insulin deficiency, which can be associated with bone disorders. In bone from type 1 diabetic rats, decreased expression of IGF-1 and IGF-1R was detected (29). Consistently, in this study, the insulin and IGF-1 signaling system in BMSCs derived from diabetic rats exhibited multiple abnormalities, including decreased expression of IR, IGF-1, IGF-1R, IRS-1 and p-ERK compared with control BMSCs. Insulin and IGF-1 signaling are important in the maintenance of normal bone metabolism. Mice lacking IR or IGF-1R in osteoblasts exhibited reduced trabecular bone and mineralization $(19,20)$. In vitro, the IR-deficient osteoblasts showed impaired proliferation, differentiation and decreased expression of Runx2 and OCN (18). In addition, IRS-1 deficiency in osteoblasts was found to impair osteoblast proliferation and differentiation (30). Therefore, abnormalities in insulin and IGF-1 signaling in diabetic BMSCs are potential contributors to reduced osteogenic potential. 
The impaired insulin and IGF-1 signaling would contribute to inhibition of the phosphorylation of ERK. Insulin and IGF-1 activate IR, IGF-1R and subsequently IRS-1, which activates the ERK pathway to regulate cell proliferation, differentiation, apoptosis and metabolism. Both insulin and IGF-1 have been shown to stimulate osteoblast proliferation and differentiation through the activation of ERK signaling pathways $(17,18)$. However, the expression levels of IR, IGF-1, IGF-1R and IRS-1 were reduced in diabetic BMSCs in our study, which led to inhibition of the phosphorylation of ERK. It is well known that the ERK pathway is crucial for the regulation of cell proliferation, osteoblast differentiation and skeletal development (31). An important function for the ERK pathway has been established in osteoblasts that involves stimulation of Runx2 (32). Thus, inhibition of the phosphorylation of ERK may also contribute to decreased proliferation and impaired osteogenic potential of diabetic BMSCs.

In summary, the present study demonstrated that the osteogenic potential of BMSCs derived from diabetic rats was impaired, which may be partially related to impaired insulin and IGF-1 signaling. These findings may advance our understanding of bone complications in diabetes. These results suggest that restoring the function of BMSCs in diabetic patients is essential to treat bone complications and that diabetic BMSCs could be an alternative cell source for bone regeneration in diabetes, including the use of additional growth factors targeting insulin and IGF-1 signaling to compensate for their deficiency.

\section{Acknowledgements}

This study was jointly supported by the National Basic Research Program of China (973 Program, No. 2012CB933604), the National Science Fund for Distinguished Young Scholars of China (No. 81225006) and the National Natural Science Foundation of China (No. 81170939).

\section{References}

1. Hofbauer LC, Brueck CC, Singh SK and Dobnig H: Osteoporosis in patients with diabetes mellitus. J Bone Miner Res 22: 1317-1328, 2007.

2. Janghorbani M, Van Dam RM, Willett WC and Hu FB: Systematic review of type 1 and type 2 diabetes mellitus and risk of fracture. Am J Epidemiol 166: 495-505, 2007.

3. Retzepi M and Donos N: The effect of diabetes mellitus on osseous healing. Clin Oral Implants Res 21: 673-681, 2010.

4. Yaturu S: Diabetes and skeletal health. J Diabetes 1: 246-254, 2009.

5. Khosla S, Westendorf JJ and Mödder UI: Concise review: Insights from normal bone remodeling and stem cell-based therapies for bone repair. Stem Cells 28: 2124-2128, 2010.

6. Mauney JR, Volloch V and Kaplan DL: Role of adult mesenchymal stem cells in bone tissue engineering applications: current status and future prospects. Tissue Eng 11: 787-802, 2005.

7. Breitbart EA, Meade S, Azad V, et al: Mesenchymal stem cells accelerate bone allograft incorporation in the presence of diabetes mellitus. J Orthop Res 28: 942-949, 2010.

8. Cramer C, Freisinger E, Jones RK, et al: Persistent high glucose concentrations alter the regenerative potential of mesenchymal stem cells. Stem Cells Dev 19: 1875-1884, 2010.

9. Khan M, Akhtar S, Mohsin S, N Khan S and Riazuddin S: Growth factor preconditioning increases the function of diabetes-impaired mesenchymal stem cells. Stem Cells Dev 20: 67-75, 2011.

10. Jin P, Zhang X, Wu Y, et al: Streptozotocin-induced diabetic rat-derived bone marrow mesenchymal cells have impaired abilities in proliferation, paracrine, antiapotosis, and myogenic differentiation. Transplant Proc 42: 2745-2752, 2010.
11. Stolzing A, Sellers D, Llewelyn O and Scutt A: Diabetes induced changes in rat mesenchymal stem cells. Cells Tissues Organs 191: 453-465, 2010.

12. Fulzele $K$ and Clemens TL: Novel functions for insulin in bone. Bone 50: 452-456, 2012.

13. AboElAsrar MA, Elbarbary NS, Elshennawy DE and Omar AM: Iusulin-like growth factor-1 cytokines cross-talk in type 1 diabetes mellitus: relationship to microvascular complications and bone mineral density. Cytokine 59: 86-93, 2012.

14. Gandhi A, Beam HA, O'Connor JP, Parsons JR and Lin SS: The effects of local insulin delivery on diabetic fracture healing. Bone 37: 482-490, 2005.

15. Thaller SR, Lee TJ, Armstrong M, Tesluk H and Stern JS: Effect of insulin-like growth factor type 1 on critical-size defects in diabetic rats. J Craniofac Surg 6: 218-223, 1995.

16. Taniguchi CM, Emanuelli B and Kahn CR: Critical nodes in signaling pathways: insights into insulin action. Nat Rev Mol Cell Biol 7: 85-96, 2006.

17. Zhang W, Shen X, Wan C, Zhao Q, Zhang L, Zhou Q and Deng L: Effects of insulin and insulin-like growth factor 1 on osteoblast proliferation and differentiation: differential signalling via Akt and ERK. Cell Biochem Funct 30: 297-302, 2012.

18. Yang J, Zhang X, Wang $\mathrm{W}$ and Liu J: Insulin stimulates osteoblast proliferation and differentiation through ERK and PI3K in MG-63 cells: cell biochemistry and function. Cell Biochem Funct 28: 334-341, 2010.

19. Fulzele K, Riddle RC, DiGirolamo DJ, et al: Insulin receptor signaling in osteoblasts regulates postnatal bone acquisition and body composition. Cell 142: 309-319, 2010.

20. Zhang M, Xuan S, Bouxsein ML, et al: Osteoblast-specific knockout of the insulin-like growth factor (IGF) receptor gene reveals an essential role of IGF signaling in bone matrix mineralization. J Biol Chem 277: 44005-44012, 2002.

21. Xian L, Wu X, Pang L, Lou M, et al: Matrix IGF-1 maintains bone mass by activation of mTOR in mesenchymal stem cells. Nat Med 18: 1095-1101, 2012.

22. Retzepi M, Lewis MP and Donos N: Effect of diabetes and metabolic control on de novo bone formation following guided bone regeneration. Clin Oral Implants Res 21: 71-79, 2010.

23. Jiang X, Zhao J, Wang S, et al: Mandibular repair in rats with premineralized silk scaffolds and BMP-2-modified bMSCs. Biomaterials 30: 4522-4532, 2009.

24. Zeng D, Xia L, Zhang W, et al: Maxillary sinus floor elevation using a tissue-engineered bone with calcium-magnesium phosphate cement and bone marrow stromal cells in rabbits. Tissue Eng Part A 18: 870-881, 2012.

25. Lü K, Zeng D, Zhang Y, et al: BMP-2 gene modified canine bMSCs promote ectopic bone formation mediated by a nonviral PEI derivative. Ann Biomed Eng 39: 1829-1839, 2011.

26. Wang XJ, Huang H, Yang F, Xia LG, Zhang WJ, Jiang XQ and Zhang FQ: Ectopic study of tissue-engineered bone complex with enamel matrix proteins, bone marrow stromal cells in porous calcium phosphate cement scaffolds, in nude mice. Cell Prolif 44: 274-282, 2011.

27. Xia L, Xu Y, Chang Q, et al: Maxillary sinus floor elevation using BMP-2 and Nell-1 gene-modified bone marrow stromal cells and TCP in rabbits. Calcif Tissue Int 89: 53-64, 2011.

28. McCabe LR: Understanding the pathology and mechanisms of type 1 diabetic bone loss. J Cell Biochem 102: 1343-1357, 2007.

29. Hie M, Iitsuka N, Otsuka T and Tsukamoto I: Insulin-dependent diabetes mellitus decreases osteoblastogenesis associated with the inhibition of Wnt signaling through increased expression of Sost and Dkk1 and inhibition of Akt activation. Int J Mol Med 28: 455-462, 2011.

30. Ogata N, Chikazu D, Kubota N, et al: Insulin receptor substrate-1 in osteoblast is indispensable for maintaining bone turnover. $\mathrm{J}$ Clin Invest 105: 935-943, 2000.

31. Stokoe D, Macdonald SG, Cadwallader K, Symons M and Hancock JF: Activation of Raf as a result of recruitment to the plasma membrane. Science 264: 1463-1467, 1994.

32. Ge C, Yang Q, Zhao G, Yu H, Kirkwood KL and Franceschi RT: Interactions between extracellular signal-regulated kinase $1 / 2$ and p38 MAP kinase pathways in the control of RUNX2 phosphorylation and transcriptional activity. J Bone Miner Res 27: 538-551, 2012. 